\title{
Aspectos histológicos dos músculos da região da escápula e do braço de anta - Tapirus terrestris - Perisodactyla, Tapiridae
}

\author{
Histological features of scapula and arm muscles of the South \\ American tapir - Tapirus terrestris - Perisodactyla, Tapiridae
}

Saulo Gonçalves Pereira*, Daniela Cristina Silva Borges, André Luiz Quagliatto Santos

Universidade Federal de Uberlândia (UFU), Uberlândia, MG, Brasil

*Autor correspondente

E-mail: saulobiologo@yahoo.com.br

\section{Resumo}

O objetivo foi descrever os aspectos histológicos do cíngulo escapular e braço de Tapirus terrestris - Linnaeus 1758. Utilizaram-se quatro animais doados ao Laboratório de Ensino e Pesquisa em Animais Silvestres da Universidade Federal de Uberlândia, após óbito sem trauma. Tapirus terrestris é um mamífero encontrado na América do Sul, e em quase todos os biomas brasileiros, porém com ínfimas informações literárias acerca de sua morfologia macro e microscópica. Desse modo, justifica-se a escolha desta espécie para o estudo, baseando-se em seus aspectos macro e micro morfológicos que, notadamente, são fundamentais para o conhecimento biológico das espécies. Foram realizados os procedimentos usuais de histologia e as colorações foram: Hematoxilina e Eosina - HE; Orceína, Reticulina e Tricômio de Masson. Histologicamente os músculos seguem o mesmo padrão dos demais mamíferos. 0 padrão histológico subsidia a relação entre estrutura corpórea e fibras musculares mais densas e fortes que dão suporte para o deslocamento em longas distâncias e para o nado eventual. Este tipo de pesquisa muito colabora com dados sobre esta espécie que é, ainda, pouco estudada.

Palavras-chave: Histologia. Escápula. Mamíferos. Morfologia descritiva.

\section{Abstract}

The aim was to describe the histological aspects of the scapular girdle and arm of Tapirus terrestris. The study was performed with four animals donated to the Laboratory for Education and Research on Wild Animals of the Federal University of Uberlândia, after death without trauma Tapirus terrestris - Linnaeus 1758 - is a mammal found in South America, and in almost all biomes, but with tiny literary information about 
macro and microscopic morphology. Thus, it justifies the choice of this species for the study, based on their macro and micro morphological features, which, notably, are fundamental to the biological knowledge of the species. Usual procedures for histology and staining were performed with hematoxylin and eosin - HE; Orcein Reticulin and Masson 's trichrome. Histologically, the muscles follow the same pattern of other mammals, the histological pattern subsidize the relationship between body structure: denser and stronger muscle fiber support for displacement over long distances and for the eventual swimming. This kind of research contributes with relevant data about this species is still poorly studied.

Keywords: Histology. Scapula. Mammals. Descriptive morphology.

\section{Introdução}

A mastofauna do Brasil tem a maior biodiversidade dos trópicos (Rocha e Dalponte, 2006); os mamíferos estão distribuídos em doze ordens: Artiodactyla, Carnívora, Cetácea, Cingulata, Chiropera, Didelphimorphia, Lagomorpha, Perisodactyla, Primates, Rodentia, Sirenia e Pilosa (Paglia et al., 2012). A anta - Tapirus terrestris (Linnaeus 1758) - é um mamífero ungulado que pertence à ordem Perissodactyla (Padilla e Dowler, 1994), que é composta por três famílias: Tapiridae (antas), Equidae (cavalos, zebras e burros) e Rhinocerotidae (rinocerontes) (Ramsay e Zainuddin, 1993). 0 gênero Tapirus tinha quatro espécies: Tapirus terrestris, Tapirus pinchaque, Tapirus bairdii e Tapirus indicus. Em 2013 foi descrita a espécie Tapirus kabomani (Cozzou et al., 2013).

Também conhecidos como "tapir", acredita-se que a palavra "anta" seja de origem árabe. Tapirus (Tupi), forma latinizada de tapir, é o nome deste animal para o povo tupi. Terrestris é aquele que vive em terra (Wilson e Reeder, 1993). São encontrados na Terra desde o Pleistoceno até os dias atuais (Holanda et al., 2007).

Os tapirídeos são macrovertebrados classificadas na ordem Perissodactyla, subordem Ceratomorpha, superfamília Tapiroides, família Tapiridae, e gênero Tapirus (Padilla e Dowler, 1994; Pereira et al., 2015). Esses mamíferos têm um número ímpar de dedos (um ou três), cada um com um casco (Hickman et al., 2001). O Tapirus terrestris (T. terrestris), diferentemente dos demais, tem quatro dedos na mão e três no pé. 0 quarto dedo do membro torácico é menos desenvolvido e eventualmente toca o solo (Quse et al., 2014).

T. terrestris é um dos maiores mamíferos das Américas e distribui-se no norte da Argentina, leste da Bolívia e Paraguai, havendo registros na Venezuela, Colômbia e Equador. No Brasil, os T. terrestris distribuem-se em quase todos os Biomas (Hershkovitz, 1954 apud Padilla e Dowler, 1994), exceto nos Pampas. São animais herbívoros, encontrados acompanhados apenas durante a época de acasalamento ou durante a amamentação (Medici, 2011). A anatomia interna e fisiologia dos tapirídeos são consideravelmente semelhantes às do cavalo doméstico e de outros perissodáctilos (Pereira et al., 2014).

Segundo Hofmann (1989) apud Heleno et al. (2011), a descrição morfológica microscópica de espécies da fauna silvestre acrescenta um enorme subsídio para o conhecimento destes, pois auxilia nas intervenções de ordem clínicocirúrgica, patológica e conservacionista. Descrições histológicas pormenorizadas contribuem para engrandecer o acervo teórico sobre as espécies, colaborando, dessa maneira, para o enriquecimento das aplicações médicas, terapêuticas e clínicas (Lima et al., 2010). 0 conhecimento histológico dos tecidos é de grande importância para o conhecimento de sua estrutura e constituição para fins patológicos e conservacionistas (Varvruk, 2012).

Dada a importância da anta para o meio ambiente como dispersor de sementes ou regulador ambiental dentro das teias alimentares, e considerando a importância do conhecimento histológico sobre a espécie para os apontamentos de ordem clínica e patológica, o objetivo desta pesquisa foi descrever 
os aspectos histológicos do cíngulo escapular e braço de Tapirus terrestris.

\section{Material e métodos}

Foram utilizadas peças anatômicas de quatro espécimes doadas por um criadouro, após óbito sem trauma, ao Laboratório de Ensino e Pesquisa em Animais Silvestres da Universidade Federal de Uberlândia (LAPAS). A pesquisa foi autorizada pelo Conselho de Ética para Utilização de Animais (CEUA) 069/12, e está de acordo com a Instrução Normativa 154/2007 do IBAMA. Esse material faz parte do acervo de pesquisas deste mesmo laboratório. As peças anatômicas foram fixadas em solução aquosa de formaldeído a 10\% (a partir da solução comercial a $3,7 \%$ ) e conservadas em cubas opacas contendo a mesma solução. Nenhum animal foi eutanasiado para esse estudo.

Para análise histológica foram realizados cortes em todos os músculos do cíngulo escapular e braço. Os fragmentos dos músculos foram submetidos aos procedimentos histológicos. Foram realizados os cortes, com espessura de $5 \mu \mathrm{m}$, em micrótomo EDGE SL-200., com as seguintes colorações: Hematoxilina e Eosina - HE para evidenciar características estruturais; Orceína para evidenciar fibras elásticas, colágeno; Reticulina para evidenciar fibras reticulares; e Tricômio de Masson para evidenciar colágeno, fibras musculares. Após a confecção das lâminas em laboratório, estas foram analisadas e fotografadas no microscópio Leica, DM 1000 na câmera Leica, Software DFU Twain 6.7.0. As espécies utilizadas na discussão foram escolhidas devido a sua disponibilidade na literatura e por serem da mesma classe que T. terrestris.

\section{Resultados e discussão}

Os tecidos musculares são responsáveis pelos movimentos corporais e são constituídos por células alongadas denominadas fibras musculares. 0 sistema esquelético forma a base estrutural dos músculos, dando base para o movimento voluntário e para a manutenção da postura. Estes músculos atuam como alavancas contráteis, que estão inseridas ao osso por meio de tendões (Stevens e Lowe, 2001). 0 tecido muscular possui uma função contrátil e considerável quantidade de filamentos citoplasmáticos de proteínas contráteis. As células musculares têm origem mesodérmica e são classificadas em três tipos: estriado esquelético, estriado cardíaco e liso (Junqueira e Carneiro, 2004). 0 músculo esquelético comporta-se como um tecido heterogêneo por expressar uma população miofibrilar diversa (Martins etal., 2007). As Figuras 1, 2, 3 e 4 apresentam os cortes histológicos de tecido muscular de alguns dos músculos de T. terrestris.
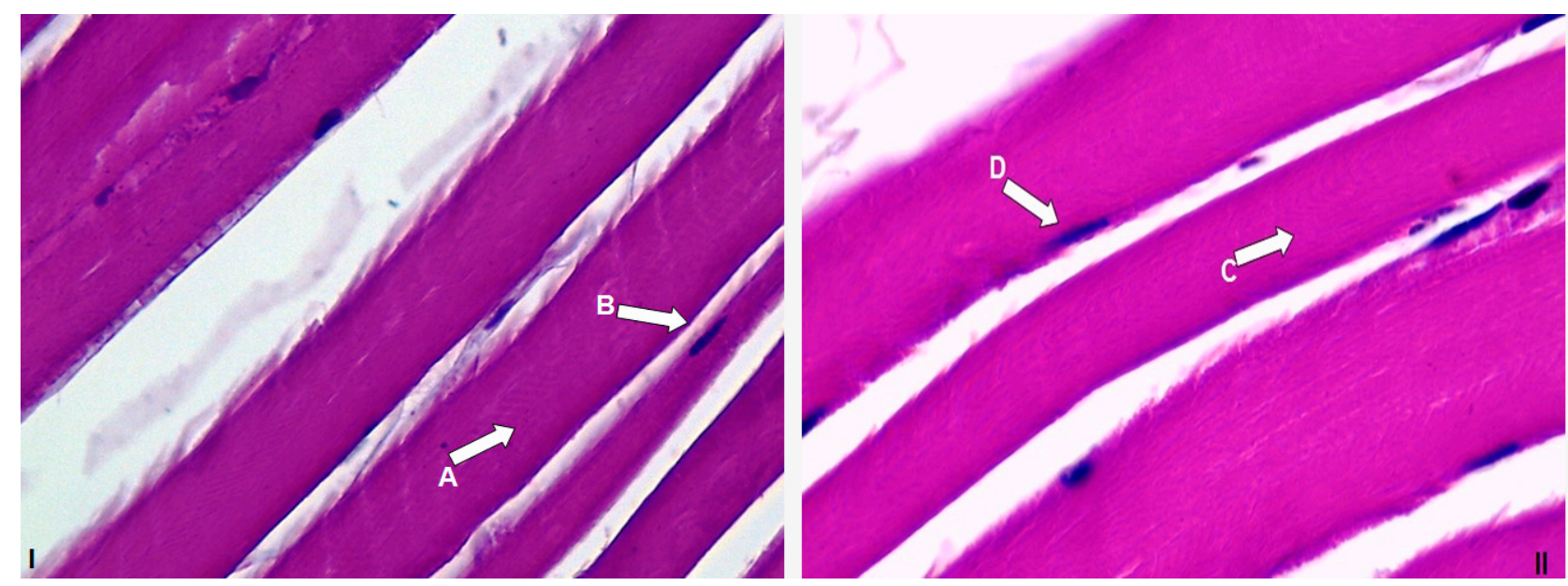

Figura 1 - Micrografia histológica em corte longitudinal corada com HE (Hematoxilina e Eosina) de Tapirus terrestris. Músculo braquial em aumento de 40x (I) e em aumento de 100x (II). Observa-se fibra muscular (A), núcleo periférico (B), fibras musculares esqueléticas com estriações longitudinais (C) e núcleos periféricos (D). 

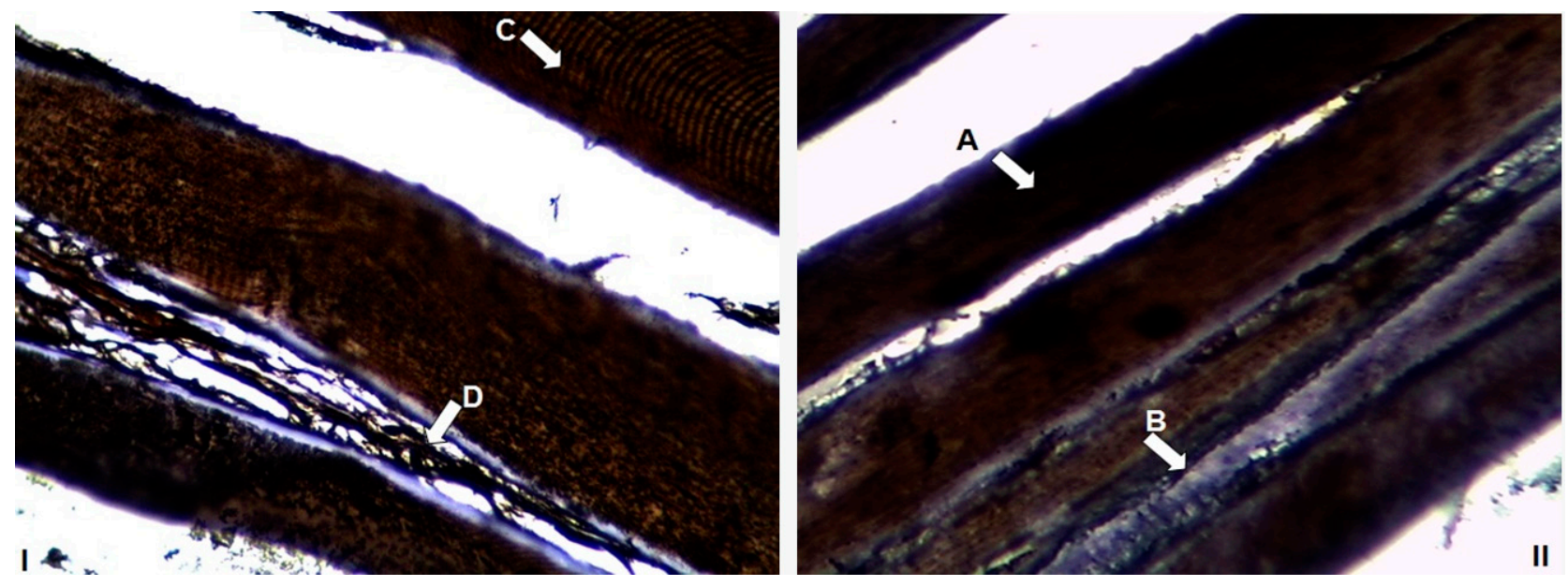

Figura 2 - Micrografia histológica em corte longitudinal do músculo bíceps braquial de Tapirus terrestris, em coloração com solução de prata amoniacal (reticulina). I - Aumento de 100x. II - Aumento de 40x. Observa-se fibra muscular (A), fibra reticular (B), fibras musculares estriadas esqueléticas com estriações longitudinais (C) e fibra reticular (D).
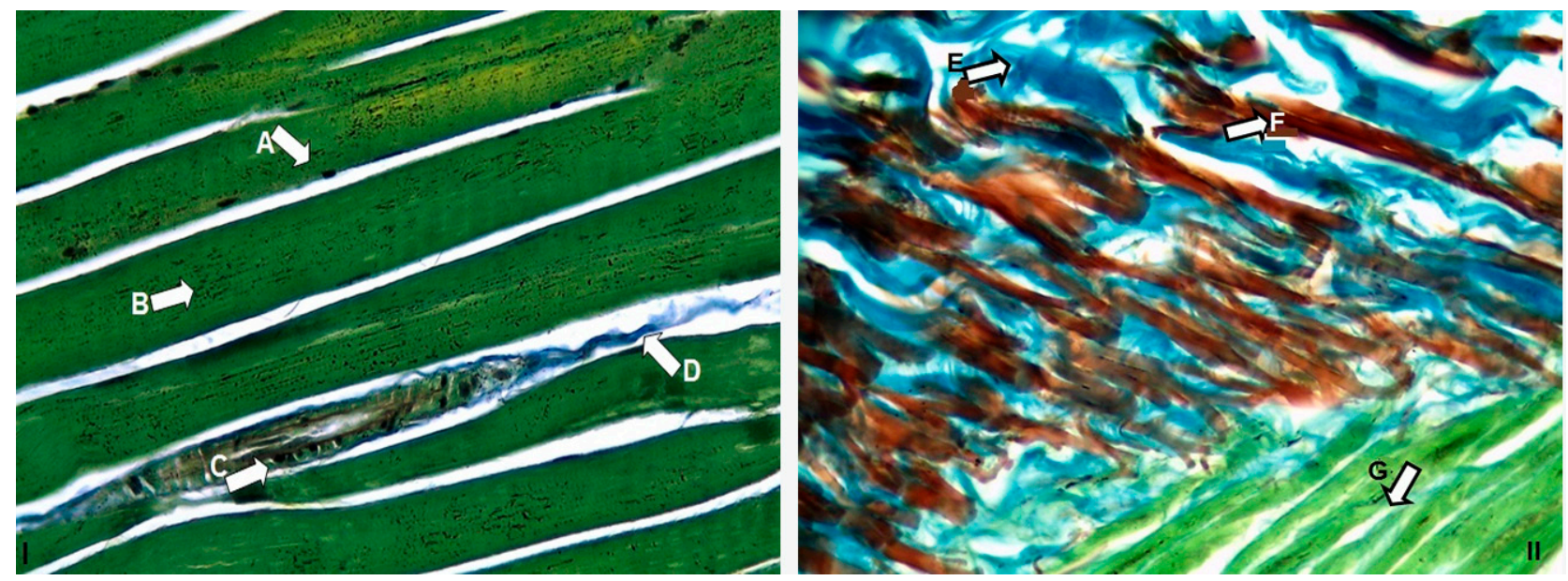

Figura 3 - : Micrografia histológica em corte longitudinal corada com orceína, a partir de material de Tapirus terrestris. I - Músculo braquial em aumento de 40x. II - Músculo subclávio em aumento de 100x. Observam-se núcleos (A), fibra estriada (B e G), vaso sanguíneo (C), colágeno (D e E) e fibras elásticas (F). 

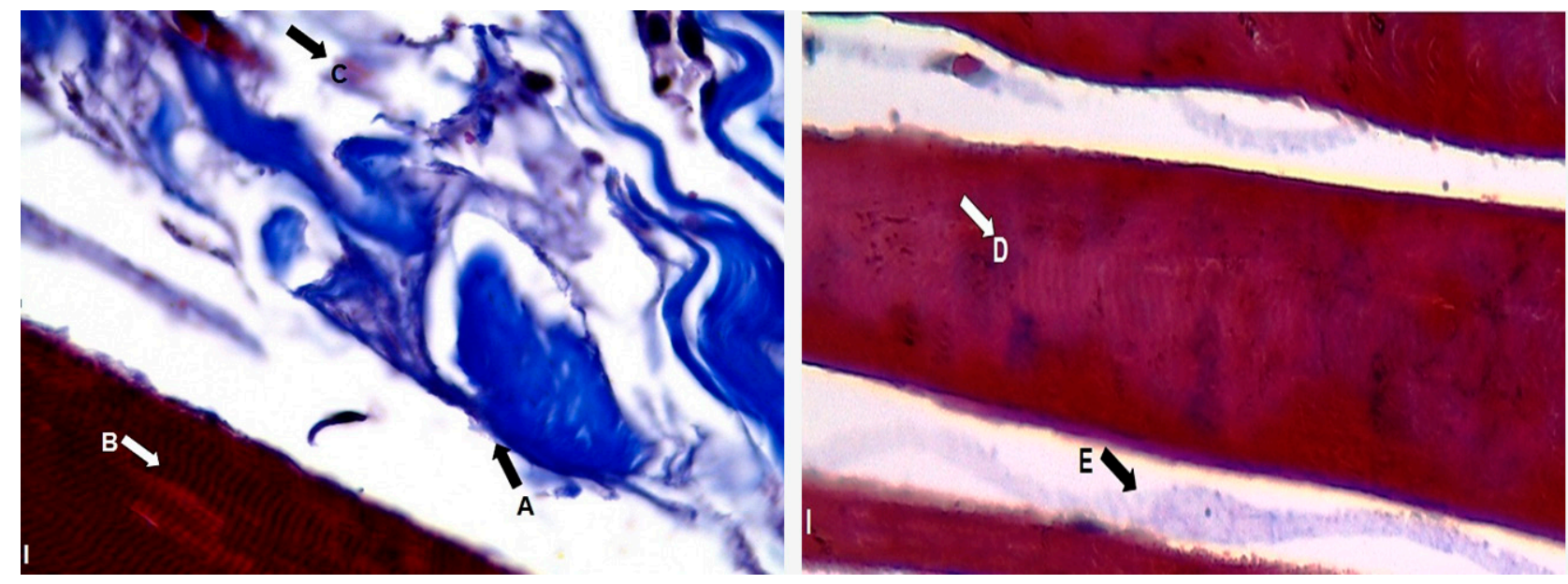

Figura 4 - Figura 4: Micrografia histológica em corte longitudinal corada com Tricômio de Masson, a partir de material de Tapirus terrestris. I - Músculo tríceps braquial em aumento de 100x. II - Músculo subescapular em aumento de 40x. Observa-se colágeno (A e E), fibra muscular estriada (B e D) e muco (C).

As fibras musculares de T. terrestris obedecem a um padrão de disposição assim como os demais mamíferos. As colorações apresentadas acima demonstram que as fibras estão dispostas na matriz como nos demais mamíferos, inclusive o ser humano (Stevens e Lowe, 2001; Junqueira e Carneiro, 2004; Kühnel, 2005).

As fibras colágenas são mais frequentes nos tecidos conjuntivos e são muito distintas e adaptadas às exigências ambientais mutáveis; é a proteína mais abundante no reino animal. Em T. terrestris são grossas e resistentes. As fibras colágenas presentes na derme conferem resistência à pele (Kühnel, 2005).

As fibras reticulares são fibras mais finas do tecido conjuntivo; são constituídas por uma proteína chamada reticulina, muito semelhante ao colágeno. Em T. terrestris, assim como em outros mamíferos, são ramificadas e formam um trançado firme que liga o tecido conjuntivo aos tecidos adjacentes (Junqueira e Carneiro, 2004).

As fibras elásticas possuem glicoproteínas, chamadas elastina, que são mais delgadas que as colágenas, podendo apresentar ramificações (Stevens e Lowe, 2001). Elas conferem elasticidade ao tecido conjuntivo frouxo, completando a resistência das fibras colágenas (Junqueira e Carneiro, 2004). Em T. terrestris estão distribuídas em todos os músculos estudados.

\section{Conclusão}

Histologicamente, os músculos de T. terrestris obedecem a um padrão de disposição das fibras estriadas, colágenas, reticulares e elásticas dos demais mamíferos. Todavia, o padrão histológico subsidia a relação entre estrutura corpórea e fibras musculares mais densas e fortes que dão suporte para o deslocamento em longas distâncias e para o nado eventual.

\section{Referências}

Heleno AR, Santos LM, Miglino MA, Peres JÁ, Guerra RR. Biometria, histologia e morfometria do sistema digestório do cachorro-do-mato (Cerdocyon thous) de vida livre. Biotemas. 2001;24(4):111-9.

Hickman Jr CP, Roberts LS, Larson A. Integrated principles of Zoology. 11를 ed. Boston: McGram-Hill; 2001. 234 p.

Holanda EC, Ferigolo J, Cartell C. Novas considerações sobre a espécie Tapirus cristatellus Winge (Mammalia, Perissodactyla), Pleistoceno superior, Estado da Bahia, Brasil. Ameghiniana. 2007;44(4):23R.

Junqueira LC, Carneiro J. Histologia Básica. 10aㅡ ed. Rio de Janeiro: Guanabara Koogan; 2004. 488 p. 
Kühnel W. 2005. Citologia, Histologia e Anatomia Microscópica: texto e atlas. 11ํㅗ ed. Porto Alegre: Artmed. $535 \mathrm{p}$

Lima FC, Santos ALQ, Lima BC, Vieira LG, Hirano LQL. Topographic anatomy of the spinal cord and vertebromedullary relationships in Mazama gouazoubira Fisher, 1814 (Artiodactyla; Cervidae). Acta sci, Biol sci. 2010;32(2):189-94.

Martins CB, Orozco C, Gomide MA, Silva G, Christovao FG, Queiroz-Neto AJC. Efeito do condicionamento atlético sobre o músculo glúteo médio de equinos Puro Sangue Arabes. Ars Vet. 2007;23(2):100-7.

Medici EP. Family Tapiridae (Tapirs). In: Wilson DE, Mittermeier RA. Handbook of the mammals of the world - Volume 2: Hoofed Mammals. Barcelona: Lynx Edicions; 2011. 886p.

Padilla M, Dowler RC. Tapirus terrestris. Mammalian Species. 1994 Dec 2;(481):1-8.

Quse V, Fernandes-Santos RC. Tapir Veterinary Manual. $2^{\circ}$ ed. Campo Grande, MS: Tapir Specialist Group; 2014. $155 p$.

Paglia AP, Fonseca GAB, Rylands AB, Herrmann G, Aguiar LMS, Chiarello AG, et al. Lista Anotada dos Mamíferos do Brasil / Annotated Checklist of Brazilian Mammals. 2 ${ }^{\underline{a}}$ Edição / 2nd Edition. Occasional Papers in Conservation Biology, no. 6. Arlington, VA: Conservation International; 2012. 76 p.

Pereira SG, Santos ALQ, Borges DCS, Souza RR, Quaioz PR. Anatomia Óssea e Muscular do Cíngulo Escapular e Braço de Tapirus Terrestris (Perissodactyla: Tapiridae). Cienc anim bras. 2015;16(2):268-78.

Ramsay CR, Zainuddin ZZ. Infectious diseases of the rhinoceros and tapir. In: Fowler ME. Zoo and Wild Animal Medicine. $3^{\circ}$ ed. Philadelphia: W.B. Saunders Company; 1993. p.459-66.

Rocha EC, Dalponte JC. Composição e caracterização da fauna de mamíferos de médio e grande porte em uma pequena reserva de cerrado em Mato Grosso, Brasil. Rev Árvore. 2006;30(4):669-78.
Stevens A, Lowe JS. Histologia humana. $2^{\circ}$ ed. Barueri: Manole; 2001. 408 p.

Varvruk JW. A importância do estudo da anatomia humana para o estudante da área de saúde. 0 anatomista. 2012;2:4-35.

Wilson DE, Reeder DE. Mammal species of the world: a taxonomic and geographic reference. $2^{\circ}$ ed. Washington, DC: Smithsonian Institution Press; 1993.

Recebido em: 23/05/2016

Received in: 05/23/2016

Aprovado em: 27/03/2017

Approved in: 03/27/2017 